\title{
Generalized conformal quantum mechanics of D0-branes
}

\author{
Donam Youm* \\ Theory Division, CERN, CH-1211, Geneva 23, Switzerland
}

(Received 19 April 1999; published 20 August 1999)

\begin{abstract}
We study the generalized conformal quantum mechanics of the probe D0-brane in the near-horizon background of the bound state of source D0-branes. We elaborate on the relationship of such a model to M theory in the light-cone frame. [S0556-2821(99)06716-8]

PACS number(s): 04.62.+v, 04.70.Dy, 11.25.Hf, 11.25.Mj
\end{abstract}

\section{INTRODUCTION}

According to the holographic principle [1-3], the bulk theory (with gravity) and the boundary theory (without gravity) are equivalent. Such correspondence gives insight into one theory from the other. Interest in the holographic principle has been revived from the recent observation [4] that the bulk gravity theory in the near-horizon geometry of brane configurations is equivalent to the boundary theory described by the corresponding world volume field theory in the decoupling limit. [The closely related previous works in Refs. [5-7] study the correspondence between the bulk theory on the anti-de Sitter (AdS) space and the boundary supersingleton field theory.] In particular, when the near-horizon geometry is the AdS space, i.e., D3-brane, M2-brane, and M5brane cases, the boundary theory is conformal. However, it is found out in Refs. [8,9] that even for other branes, whose near-horizon geometry is not the AdS type and therefore the corresponding boundary theory is not a "genuine" conformal theory, one can still define "generalized" conformal theory.

The symmetry group of such a generalized conformal theory can be manifestly seen in the bulk theory in the socalled "dual" frame $[10,6,7,11-13]$. The dual frame is regarded as a preferred frame for supergravity probes in the near-horizon background of the source branes. In this frame, the near-horizon geometry of a $p$-brane supergravity solution takes the $\mathrm{AdS}_{p+2} \times \mathrm{S}^{n}$ form. The $\mathrm{SO}(p+1,2)$ isometry of the $\mathrm{AdS}_{p+2}$ part is realized in the boundary theory as the "generalized" conformal symmetry of Refs. [8,9]. The dual frame is also called a "holographic" frame, since an UV-IR connection between the bulk and the boundary theory is manifest in this frame.

In this paper, we study the D0-brane case of such a generalized conformal theory. The D0-brane is particularly interesting for its relevance to M theory. An important discovery of the $\mathrm{M}$ (atrix) model $^{1}$ is that difficult problems of quantum $\mathrm{M}$ theory are reduced to nonrelativistic quantum mechanics. Originally, it is conjectured [16] that M theory in the infinite momentum frame (IMF) [17] is exactly described by the $U(\infty) D=1$ super-Yang-Mills (SYM) theory, which is the world volume theory of the bound state of an infinite

\footnotetext{
*Email address: Donam.Youm@cern.ch

${ }^{1}$ This model was proposed [14] long ago as $N=16$ supersymmetric gauge quantum mechanics. See also Ref. [15].
}

number $(N \rightarrow \infty)$ of D0-branes. The $D=1 \mathrm{U}(N)$ SYM theory, which is the supersymmetric matrix model description of the supermembrane theory [18], is nothing but the maximally supersymmetric $\mathrm{U}(N)$ Yang-Mills theory dimensionally reduced from $9+1$ to $0+1$ dimensions (i.e., $N$ $\times N$ Hermitian matrix quantum mechanics). It is further conjectured [19] that the equivalence of (M)atrix theory to $M$ theory is also valid for a finite $N$. The conjecture states that $\mathrm{M}$ theory compactified on a lightlike circle with finite momentum along the circle is exactly described by a $\mathrm{U}(N)$ matrix theory. The quantization of such a theory is called the discrete light-cone quantization (DLCQ). One can think of this lightlike circle as a small spacelike circle that is boosted by a large amount [20]. Just like M theory in the IMF, M theory in the light-cone frame (LCF) is described purely by D0-branes with positive momentum and has Galilean invariance in the transverse space [19]. Therefore, M theory on a lightlike circle can be viewed as a theory of a finite number of D0-branes in the low velocity (nonrelativistic) limit.

This idea can also be understood from the bulk-boundary duality as follows. When compactified on a lightlike circle, the supergravity M-wave solution (viewed as the supergraviton with the momentum number $N$ ) becomes the nearhorizon limit of the supergravity solution for $N$ coinciding D0-branes [21]. Since M theory in the LCF in the supergravity solution level is related to the near-horizon limit of the supergravity D0-brane solution, by the generalized AdS/ conformal-field-theory(CFT) duality $[22,8,9] \mathrm{M}$ theory in the LCF has to be related to the boundary theory of the bound state of $N$ D0-branes. This boundary theory is the maximally supersymmetric $\mathrm{SU}(N)$ Yang-Mills theory dimensionally reduced from $9+1$ to $0+1$ dimensions, as stated in the (M)atrix theory conjecture.

In this paper, we view the nonrelativistic (matrix) quantum mechanics of bound states of D0-branes as a system of the probe D0-brane moving in the background of a large number of source D0-brane bound states. This view of the (M)atrix model is also taken in Ref. [21], which reproduces the (M)atrix model graviton-graviton scattering calculation from the effective action for a probe moving in the background of the M-wave supergravity solution. It is further shown in Refs. [8,9] that the Dirac-Born-Infeld (DBI) action for a radially moving probe D0-brane in the background of the source D0-brane bound state can also be determined by imposing the generalized conformal symmetry of the boundary theory, which is just the $D=1 \mathrm{U}(N)$ SYM theory de- 
scribing $\mathrm{M}$ theory in the LCF.

The quantum mechanics of the probe D0-brane in the near-horizon background of the source D0-brane is reminiscent of the conformal quantum mechanics of a charged test particle moving in the near-horizon background of a $D=4$ extreme Reissner-Nordström black hole studied in Ref. [23]. The radial motion of such a test particle is described by the relativistic conformal quantum mechanics with the $\operatorname{SL}(2, \mathbf{R})$ $\cong \mathrm{SU}(1,1)$ symmetry. This is a generalized version of (nonrelativistic) conformal mechanics studied in Refs. [24-26]. One can view such generalized conformal mechanics of the radial motion of the test particle as the boundary conformal field theory counterpart of the bulk gravity theory in the near-horizon $\mathrm{AdS}_{2}$ geometry of the extreme ReissnerNordström black hole, since the $\mathrm{SO}(1,2) \cong \mathrm{SU}(1,1)$ isometry of the $\mathrm{AdS}_{2}$ space is realized as the symmetry of the dynamics of the test particle in spacetime with one lower dimension. Since the near-horizon geometry of the source D0brane supergravity solution in the dual frame is $\operatorname{AdS}_{2} \times \mathrm{S}^{8}$, one would expect that the dynamics of the probe D0-brane in this background has $\operatorname{SL}(2, \mathbf{R}) \cong \mathrm{SO}(2,1)$ symmetry. In the D0-brane case, since this symmetry does not extend to the genuine conformal symmetry of the boundary theory but only to the so-called generalized conformal symmetry $[8,9]$ as pointed out in the above, the quantum mechanics of the probe D0-brane will have a generalized conformal symmetry.

The paper is organized as follows. In Sec. II, we summarize the properties of the near-horizon geometry of the supergravity D0-brane solution. In Sec. III, we study the "generalized" conformal quantum mechanics of D0-branes, elaborating its relation to $\mathrm{M}$ theory as pointed out in the above.

\section{D0-BRANE SOLUTION IN THE NEAR-HORIZON REGION}

In this section, we survey aspects of the D0-brane supergravity solution and its near-horizon geometry, illuminating their relations.

The string-frame type-IIA effective supergravity action for the D0-brane solution is given by

$$
\begin{aligned}
S= & \frac{1}{16 \pi G_{10}} \int d^{10} x \sqrt{-G^{s t r}}\left[e^{-2 \phi}\left(\mathcal{R}^{s t r}+4 \partial_{M} \phi \partial^{M} \phi\right)\right. \\
& \left.-\frac{1}{2 \times 2 !} F_{M N} F^{M N}\right],
\end{aligned}
$$

where $G_{10}$ is the ten-dimensional gravitational constant, $\mathcal{R}^{\text {str }}$ and $G^{s t r}$ are the Ricci scalar and the determinant for the string-frame metric tensor $G_{M N}^{s t r}, \phi$ is the dilaton in the Neveu-Schwarz-Neveu-Schwarz (NS-NS) sector, and $F_{M N}$ is the field strength for the Ramond-Ramond (RR) one-form potential $A_{M}$ that D0-branes couple to.

The supergravity solution for the D0-brane has the following form:

$$
d s_{s t r}^{2}=G_{M N}^{s t r} d x^{M} d x^{N}=-H^{-1 / 2} d t^{2}+H^{1 / 2}\left(d x_{1}^{2}+\cdots+d x_{9}^{2}\right),
$$

$$
e^{\phi}=g_{s} H^{3 / 4}, \quad A_{t}=-g_{s}^{-1} H^{-1}, \quad H=1+\frac{Q}{r^{7}} \equiv 1+\left(\frac{\mu}{r}\right)^{7},
$$

where $g_{s}$ is the string coupling constant, which is just the vacuum expectation value or the asymptotic value of the dilaton $e^{\phi}$. Here, the D0-brane charge $Q$ is related to the string theory quantities as

$$
Q=\left(\alpha^{\prime}\right)^{7 / 2} g_{s} N
$$

where $\alpha^{\prime}$ is related to the string length scale $l_{s}$ as $l_{s}=\sqrt{\alpha^{\prime}}$ and $N$ is the number of the D0-branes. By expanding the DBI action for the D0-brane to the lowest order in $\alpha^{\prime}$, one finds the following relation of the string theory quantities to the Yang-Mills gauge coupling $g_{Y M}$ :

$$
g_{Y M}^{2}=\frac{g_{s}}{\left(\alpha^{\prime}\right)^{3 / 2}} .
$$

Therefore, the constant $Q$ in the harmonic function is rewritten in terms of the SYM quantities as $Q=\mu^{7}=\left(\alpha^{\prime}\right)^{5} g_{Y M}^{2} N$.

In order to decouple the massive string modes (whose masses are proportional to $1 / \alpha^{\prime}$ ) and the gravity modes [whose strength goes as $g_{s}^{2}\left(\alpha^{\prime}\right)^{4}$ ] from the massless open string modes, which describe the Yang-Mills theory, one has to take $\alpha^{\prime} \rightarrow 0$, while keeping $g_{Y M}$ as a finite constant, which means for the D0-brane case $g_{s} \rightarrow 0$ as well [cf. Eq. (4)]. Furthermore, the above metric (2) can be regarded as the gravitational field felt by a probe D0-brane in the background of a collection of $N$ numbers of source D0-branes with the radial coordinate $r$ being interpreted as the distance between and probe and source D0-branes [27]. So in order to keep the mass $m_{\text {string }}=r / \alpha^{\prime}$ of the state of open string, which stretches between the source and the probe, finite, one has also take the limit $r \rightarrow 0$ while keeping the following combination as a finite constant:

$$
U \equiv \frac{r}{\alpha^{\prime}}
$$

thereby going to the near-horizon region of the supergravity solution (2). This combination also corresponds to the conventional super-Yang-Mills scalar $\Phi^{I}=X^{I} / l_{s}^{2}$, whose vacuum expectation value sets the energy scale. In this decoupling limit, the supergravity solution (2) takes the following form:

$$
\begin{aligned}
d s_{s t r}^{2} & =-\left(\frac{r}{\mu}\right)^{7 / 2} d t^{2}+\left(\frac{\mu}{r}\right)^{7 / 2}\left(d r^{2}+r^{2} d \Omega_{8}^{2}\right) \\
& =\alpha^{\prime}\left[-\frac{U^{7 / 2}}{g_{Y M} \sqrt{N}} d t^{2}+\frac{g_{Y M} \sqrt{N}}{U^{7 / 2}}\left(d U^{2}+U^{2} d \Omega_{8}^{2}\right)\right], \\
e^{\phi} & =g_{s}\left(\frac{\mu}{r}\right)^{21 / 4}=g_{Y M}^{2}\left(\frac{g_{Y M}^{2} N}{U^{7}}\right)^{3 / 4},
\end{aligned}
$$




$$
A_{t}=g_{s}^{-1}\left(\frac{r}{\mu}\right)^{7}=\frac{\sqrt{\alpha^{\prime}}}{g_{Y M}^{2}} \frac{U^{7}}{g_{Y M}^{2} N}
$$

The above supergravity solution (6) can be trusted when the spacetime curvature $\mathcal{R} \sim U^{3} /\left(g_{Y M}^{2} N\right)$ is much smaller than the string scale $\left(\alpha^{\prime}\right)^{-1}$ and the string coupling $e^{\phi}$ is very small, leading to the following constraints on $U$ and the original radial coordinate $r$ :

$$
\begin{aligned}
& g_{Y M}^{2 / 3} N^{1 / 7} \ll U \ll g_{Y M}^{2 / 3} N^{1 / 3}, \\
& \sqrt{\alpha^{\prime}} g_{s}^{1 / 3} N^{1 / 7} \ll r \ll \sqrt{\alpha^{\prime}} g_{s}^{1 / 3} N^{1 / 3} .
\end{aligned}
$$

On the other hand, the near-horizon condition $r \ll \mu=Q^{1 / 7}$ is expressed in terms of string theory quantities as $r$ $\ll \sqrt{\alpha^{\prime}}\left(g_{s} N\right)^{1 / 7}$. So for sufficiently large $N$ and small $g_{s}$, the supergravity solution (6) can be trusted in the overlapping region of $U$.

Note that the near-horizon geometry of the D0-brane supergravity solution in the string frame is not $\mathrm{AdS}_{2} \times \mathrm{S}^{8}$, since when the metric is expressed in the suggestive form

$$
d s_{s t r}^{2}=\alpha^{\prime}\left[-\frac{U^{2}}{\sqrt{\rho_{0}}} d t^{2}+\sqrt{\rho_{0}} \frac{d U^{2}}{U^{2}}+\sqrt{\rho_{0}} d \Omega_{8}^{2}\right],
$$

the radius $\rho_{0} \equiv Q /\left[\left(\alpha^{\prime}\right)^{5} U^{3}\right]$ of the would-be AdS space depends on the coordinate $U$.

However, with the suitable choice of frame, called the "dual" frame $[10,6,7,13]$, the spacetime metric in the nearhorizon region takes the $\mathrm{AdS}_{2} \times \mathrm{S}^{8}$ form. Namely, if one applies the Weyl transformation ${ }^{2}$ to the metric as $G_{M N}^{s t r}$ $\rightarrow G_{M N}^{d u a l}=e^{-2 \phi / 7} G_{M N}^{s t r}$, then the metric in Eq. (6) transforms into

$$
\begin{aligned}
d s_{\text {dual }}^{2} & =G_{M N}^{\text {dual }} d x^{M} d x^{N} \\
& =g_{s}^{-2 / 7}\left[-\left(\frac{r}{\mu}\right)^{5} d t^{2}+\left(\frac{\mu}{r}\right)^{2} d r^{2}+\mu^{2} d \Omega_{8}^{2}\right] \\
& =N^{2 / 7} \alpha^{\prime}\left[-\frac{U^{5}}{g_{Y M}^{2} N} d t^{2}+\frac{d U^{2}}{U^{2}}+d \Omega_{8}^{2}\right] .
\end{aligned}
$$

By further redefining the radial coordinate as $\bar{r}$ $=\left(\frac{25}{4} g_{s}^{4 / 7} \mu^{3}\right)^{-1 / 2} r^{5 / 2}$ or $u=\left(\frac{25}{4} g_{Y M}^{2} N^{3 / 7}\right)^{-1 / 2} U^{5 / 2}$, one can bring the metric to the following standard $\mathrm{AdS}_{2} \times \mathrm{S}^{8}$ form in the horospherical coordinates:

\footnotetext{
${ }^{2}$ In this paper, we do not include a factor involving $N$ in the Weyl transformation of the metric, unlike Ref. [13], so that the metric in the standard AdS form in the horospherical coordinates has an explicit dependence on $N$.
}

$$
\begin{aligned}
d s_{\text {dual }}^{2} & =-\left(\frac{5 g_{s}^{1 / 7}}{2 \mu}\right)^{2} \bar{r}^{2} d t^{2}+\left(\frac{2 \mu}{5 g_{s}^{1 / 7}}\right)^{2} \frac{d \bar{r}^{2}}{\bar{r}^{2}}+\left(\frac{\mu}{g_{s}^{1 / 7}}\right)^{2} d \Omega_{8}^{2} \\
& =\alpha^{\prime}\left[-\left(\frac{5}{2 N^{1 / 7}}\right)^{2} u^{2} d t^{2}+\left(\frac{2 N^{1 / 7}}{5}\right)^{2} \frac{d u^{2}}{u^{2}}+N^{2 / 7} d \Omega_{8}^{2}\right],
\end{aligned}
$$

where $u=\bar{r} / \alpha^{\prime}$. In the dual frame, in which the metric in the near horizon takes the above AdS form, the effective action (1) takes the following form:

$$
\begin{aligned}
S= & \frac{1}{16 \pi G_{10}} \int d^{10} x \sqrt{-G^{\text {dual }}}\left[e^{-6 \phi / 7}\left(\mathcal{R}^{\text {dual }}+\frac{16}{49} \partial_{M} \phi \partial^{M} \phi\right)\right. \\
& \left.-\frac{1}{4} e^{6 \phi / 7} F_{M N} F^{M N}\right] .
\end{aligned}
$$

On the other hand, one can view the D0-brane solution (2) as being magnetically charged under the Hodge dual field strength to the field strength of the one-form potential in the RR sector. Namely, the supergravity solution (2) solves the equations of motion of the following effective action and is magnetically charged under the seven-form potential $A_{M_{1} \cdots M_{7}}$ :

$$
\begin{aligned}
S^{\prime}= & \frac{1}{16 \pi G_{10}} \int d^{10} x \sqrt{-G^{s t r}}\left[e^{-2 \phi}\left(\mathcal{R}^{s t r}+4 \partial_{M} \phi \partial^{M} \phi\right)\right. \\
& \left.-\frac{1}{2 \times 8 !} F_{M_{1} \cdots M_{8}} F^{M_{1} \cdots M_{8}}\right]
\end{aligned}
$$

where $F_{M_{1} \cdots M_{8}}$ is the field strength of the seven-form potential $A_{M_{1} \cdots M_{7}}$. In the dual frame with the spacetime metric $G_{M N}^{\text {dual }}=e^{-2 \phi / 7} G_{M N}^{s t r}$, the action (13) takes the following form [13]:

$$
\begin{aligned}
S^{\prime}= & \frac{1}{16 \pi G_{10}} \int d^{10} x \sqrt{-G^{d u a l}} e^{-6 \phi / 7}\left[\mathcal{R}^{d u a l}+\frac{16}{49} \partial_{M} \phi \partial^{M} \phi\right. \\
& \left.-\frac{1}{2 \times 8 !} F_{M_{1} \cdots M_{8}} F^{M_{1} \cdots M_{8}}\right] .
\end{aligned}
$$

As in Ref. [13], if one properly includes the factor involving $N$ in the Weyl transformation of the metric, i.e., $G_{M N}^{s t r}$ $\rightarrow G_{M N}^{d u a l}=\left(N e^{\phi}\right)^{-2 / 7} G_{M N}^{s t r}$; then the new radial coordinate $u$ (with which the near-horizon metric takes the AdS form in the horospherical coordinates) is related to $U$ as $u$ $=\frac{2}{5} U^{5 / 2} /\left(g_{Y M} N^{1 / 2}\right)$. This is reminiscent of the holographic UV-IR connection between the bulk and boundary theories $[28,29]$, if one identifies $u$ with the energy scale $E$ of the boundary theory [13].

In this case, the effective action in the dual frame takes the following form [13]: 


$$
\begin{aligned}
S^{\prime}= & \frac{N^{2}}{16 \pi G_{10}} \int d^{10} x \sqrt{-G^{\text {dual }}}\left(N e^{\phi}\right)^{-6 / 7}\left[\mathcal{R}^{\text {dual }}\right. \\
& \left.+\frac{16}{49} \partial_{M} \phi \partial^{M} \phi-\frac{1}{2 \times 8 !} \frac{1}{N^{2}} F_{M_{1} \cdots M_{8}} F^{M_{1} \cdots M_{8}}\right]
\end{aligned}
$$

The near-horizon form of the D0-brane solution of the associated equations of motion is

$$
\begin{aligned}
d s_{d u a l}^{2} & =-\frac{u^{2}}{u_{0}^{2}} d t^{2}+u_{0}^{2} \frac{d u^{2}}{u^{2}}+d \Omega_{8}^{2}, \\
e^{\phi} & =\frac{1}{N}\left(g_{Y M}^{2} N\right)^{7 / 10}\left(\frac{u}{u_{0}}\right)^{-21 / 10}, \\
F_{8} & =7 N \operatorname{vol}\left(S^{8}\right),
\end{aligned}
$$

where $u_{0}=2 / 5$. From this solution, one can see that there is the Freund-Rubin compactification [30] on $S^{8}$ of the $D=10$ action (15) to the following two-dimensional effective gauged supergravity action [13]:

$$
S^{\prime}=N^{2} \int d^{2} x \sqrt{-g}\left(N e^{\phi}\right)^{-6 / 7}\left[\mathcal{R}+\frac{16}{49} \partial_{\mu} \phi \partial^{\mu} \phi+\frac{63}{2}\right] \text {. }
$$

The near-horizon D0-brane supergravity solution (16) is reduced under this $S^{8}$ compactification to a domain solution [31-33], which is supported by the cosmological term in the action (17).

\section{GENERALIZED CONFORMAL MECHANICS OF D0-BRANES}

We consider a probe D0-brane with mass $m$ and charge $q$ moving in the near-horizon geometry of the source $N$ D0brane bound state. The gravitational field felt by the probe D0-brane in the string [dual] frame is given by Eqs. (6) and (9) [Eqs. (10) and (11)]. Here, once again, $r$ is the radial distance between the source and the probe D0-branes. The probe D0-brane moves with the ten-momentum $p=\left(p_{M}\right)$ $=\left(p_{t}, p_{1}, \ldots, p_{9}\right)$ and its time component is the (staticgauge) Hamiltonian $H=-p_{t}$. The expression for the Hamiltonian of the probe D0-brane in the near-horizon background of the source D0-brane can be obtained by solving the massshell constraint of the probe D0-brane.

Unlike the case of Ref. [23], which studies a charged particle in Einstein-Maxwell theory, the probe D0-brane satisfies the mass-shell constraint which is different from the ordinary constraint $0=(p-q A)^{2}+m^{2}=G^{M N}\left(p_{M}\right.$ $\left.-q A_{M}\right)\left(p_{N}-q A_{N}\right)+m^{2}$. This is due to the nontrivial dilaton field that is present for the D0-brane solution. So here we rederive the mass-shell condition for the case of D0-branes. The action for the probe D0-brane with the mass $m$ and the charge $q$ moving in the background of the source D0-brane has the following form:

$$
S=\int d \tau L=\int d \tau\left(m e^{-\phi} \sqrt{-G_{M N}^{s t r} \dot{x}^{M} \dot{x}^{N}}-q \dot{x}^{M} A_{M}\right),
$$

where $\mathrm{v}^{M}=\dot{x}^{M} \equiv d x^{M} / d \tau$ is the ten-velocity of the probe D0brane. Note that, since we choose the static gauge for the action, the world line time $\tau$ and the target-space time $t$ are set to be equal. Once again, $G_{M N}^{s t r}$ and $A_{M}$ are the fields produced by the source D0-brane. Note that in this action the metric $G_{M N}^{s t r}$ is in the string frame. The (generalized) momentum conjugate to $x^{M}(\tau)$ is

$$
P_{M}=-\frac{\delta L}{\delta \dot{x}^{M}}=\frac{m e^{-\phi} \dot{x}_{M}}{\sqrt{-G_{M N}^{s t r} \dot{x}^{M} \dot{x}^{N}}}+q A_{M} .
$$

As usual, $p_{M}=m \dot{x}_{M} / \sqrt{-G_{M N}^{s t r} \dot{x}^{M} \dot{x}^{N}}$ is the ordinary tenmomentum of the D0-brane. From this, one obtains the following mass-shell constraint for the probe D0-brane in the string-frame background of the probe D0-brane:

$$
G^{\text {str } M N}\left(P_{M}-q A_{M}\right)\left(P_{N}-q A_{N}\right)+m^{2} e^{-2 \phi}=0 .
$$

To obtain the expression for the Hamiltonian $H=-P_{t}$ for the probe D0-brane mechanics, we solve this mass-shell constraint (20). We consider the following general spherically symmetric $D=10$ metric ansatz:

$$
\begin{aligned}
G_{M N} d x^{M} d x^{N}= & -A(r) d t^{2}+B(r) d r^{2}+C(r) d \Omega_{8}^{2} \\
= & -A(r) d t^{2}+B(r) d r^{2}+C(r)\left[d \theta^{2}\right. \\
& +\cos ^{2} \theta d \psi_{1}^{2}+\cos ^{2} \theta \cos ^{2} \psi_{1} d \psi_{2}^{2} \\
& \left.+\cos ^{2} \theta \cos ^{2} \psi_{1} \cos ^{2} \psi_{2} d \psi_{3}^{2}+\mu_{i}^{2} d \phi_{i}^{2}\right],
\end{aligned}
$$

where

$$
\begin{gathered}
\mu_{1}=\sin \theta, \quad \mu_{2}=\cos \theta \sin \psi_{1}, \quad \mu_{3}=\cos \theta \cos \psi_{1} \sin \psi_{2} \\
\mu_{4}=\cos \theta \cos \psi_{1} \cos \psi_{2} \sin \psi_{3}
\end{gathered}
$$

Then, the expression for the Hamiltonian $H$ takes the following form:

$$
H=\frac{P_{r}^{2}}{2 f}+\frac{g}{2 f}
$$

where

$$
\begin{aligned}
f \equiv & \frac{1}{2} A^{-1 / 2} B e^{-\phi}\left[\sqrt{m^{2}+e^{2 \phi}\left(P_{r}^{2}+B C^{-1} \vec{L}^{2}\right) / B}\right. \\
& \left.+q A^{-1 / 2} A_{t} e^{\phi}\right], \\
g \equiv & B e^{-2 \phi}\left[\left(m^{2}-q^{2} A^{-1} A_{t}^{2} e^{2 \phi}\right)+C^{-1} e^{2 \phi} \vec{L}^{2}\right] .
\end{aligned}
$$

Here, $A_{t}$ is the time component of the one-form field $A_{M}$ of the near-horizon source D0-brane supergravity solution (6) and $\vec{L}^{2}$ is the angular momentum operator of the probe D0brane given by 


$$
\begin{aligned}
\vec{L}^{2}= & P_{\theta}^{2}+\frac{P_{\psi_{1}}^{2}}{\cos ^{2} \theta}+\frac{P_{\psi_{2}}^{2}}{\cos ^{2} \theta \cos ^{2} \psi_{1}}+\frac{P_{\psi_{3}}^{2}}{\cos ^{2} \theta \cos ^{2} \psi_{1} \cos ^{2} \psi_{2}} \\
& +\sum_{i=1}^{4} \frac{P_{\phi_{i}}^{2}}{\mu_{i}^{2}} .
\end{aligned}
$$

First, we consider the probe D0-brane moving in the string-frame near-horizon background of the source D0brane. In the original work [23] of the superconformal mechanics of a test charged particle in the near-horizon geometry of the Reissner-Nordström black hole, it was necessary to redefine the radial coordinate so that the metric components $A$ and $B$ in Eq. (21) satisfy the relation $A=B^{2}$ for the purpose of setting the factor $A^{-1 / 2} B$ in Eq. (23) equal to 1 . However, in the D0-brane case, as we will see, it is more convenient to work with the original form (6) of the nearhorizon metric, since the expression for the Hamiltonian becomes simpler in the original radial coordinate.

By substituting the string-frame near-horizon metric into the general formulas (23) and (24), one obtains the following Hamiltonian for the probe D0-brane in the string-frame nearhorizon background of the source D0-brane bound state:

$$
H=\frac{P_{r}^{2}}{2 f}+\frac{g}{2 f},
$$

where $f$ and $g$ are given by

$$
\begin{aligned}
& f=\frac{1}{2} g_{s}^{-1}\left[\sqrt{m^{2}+g_{s}^{2}\left(\frac{\mu}{r}\right)^{7}\left(P_{r}^{2}+\frac{\vec{L}^{2}}{r^{2}}\right)}+q\right], \\
& g=g_{s}^{-2}\left(\frac{\mu}{r}\right)^{-7}\left(m^{2}-q^{2}\right)+\frac{\vec{L}^{2}}{r^{2}},
\end{aligned}
$$

or in terms of the SYM theory variables

$$
H=\frac{P_{U}^{2}}{2 f}+\frac{g}{2 f}
$$

where $f$ and $g$ are given by

$$
\begin{aligned}
& f=\frac{1}{2} \alpha^{\prime 1 / 2} g_{Y M}^{-2}\left[\sqrt{m^{2}+\frac{\alpha^{\prime-1} g_{Y M}^{6} N}{U^{7}}\left(P_{U}^{2}+\frac{\vec{L}^{2}}{U^{2}}\right)}+q\right] \\
& g=\left(\frac{\alpha^{\prime-1} g_{Y M}^{6} N}{U^{7}}\right)^{-1}\left(m^{2}-q^{2}\right)+\frac{\vec{L}^{2}}{U^{2}}
\end{aligned}
$$

It is interesting that the term $A^{-1} A_{t}^{2} e^{2 \phi}$ in Eq. (24) becomes 1 for the near-horizon D0-brane solution (6). So the expressions for $f$ and $g$ become greatly simplified. And, in particular, in the extreme limit $(m-q \rightarrow 0)$ of the probe D0-brane, the first term in $g$ drops out. This also generally holds for any dilatonic 0-brane supergravity solutions.

Just as in the case of the test charged particle in the Reissner-Nordström black hole background, the mechanics of the probe D0-brane has $\operatorname{SL}(2, \mathbf{R})$ symmetry with the following generators:

$$
H=\frac{P_{r}^{2}}{2 f}+\frac{g}{2 f}, \quad K=-\frac{1}{2} f r^{2}, \quad D=\frac{1}{2} r P_{r},
$$

where the Hamiltonian $H$ generates the time translation, $K$ generates the special conformal transformation, and $D$ generates the scale transformation or the dilatation. These generators satisfy the following $\operatorname{SL}(2, \mathbf{R})$ algebra:

$$
[D, H]=H, \quad[D, H]=-K, \quad[H, K]=2 D .
$$

This is the D0-brane generalization of conformal quantum mechanics studied in Refs. [24,26,23].

In fact, the near-horizon solution (6), when uplifted as a solution of the 11-dimensional gravity (i.e., the 11dimensional plane-wave solution), is invariant under the $\mathrm{SU}(1,1) \cong \mathrm{SL}(2, \mathbf{R})$ isometry ${ }^{3}$ generated by the scale transformation $\delta_{D}$, the special coordinate transformation $\delta_{K}$, and the time translation $\delta_{H}$ [8]. Under the time translation, $\delta_{H} t$ $=1, \delta_{H} U=0$, and $\delta_{H} g_{s}=0$. Under the special coordinate transformation, $\delta_{K} t=-\left(t^{2}+k g_{Y M}^{2} / U^{5}\right), \quad \delta_{K} U=2 t U$, and $\delta_{K} g_{s}=6 t g_{s}$. Finally, under the dilatation, $\delta_{D} t=-t, \delta_{D} U$ $=U$, and $\delta_{D} g_{s}=3 g_{s}$. And $A_{t}$ transforms as a conformal field of dimension 1 . These infinitesimal transformations satisfy the following $\operatorname{SL}(2, \mathbf{R})$ algebra just like the symmetry generators (30) of the probe D0-brane mechanics:

$$
\left[\delta_{D}, \delta_{H}\right]=\delta_{H}, \quad\left[\delta_{D}, \delta_{K}\right]=-\delta_{K}, \quad\left[\delta_{H}, \delta_{K}\right]=2 \delta_{D} \text {. }
$$

Note that the string coupling $g_{s}$ changes under the dilatation and the special coordinate transformation, and especially $g_{s}$ becomes time dependent after the special coordinate transformation is applied. Thereby, this $\operatorname{SL}(2, \mathbf{R})$ isometry of the near-horizon geometry does not extend to a conformal symmetry of the complete supergravity solution. The corresponding boundary theory, i.e., $(0+1)$-dimensional SYM matrix quantum mechanics, has the same $\operatorname{SL}(2, \mathbf{R})$ symmetry, but the string coupling $g_{s}$ also transforms under this symmetry, unlike the case of D3-branes. However, as noted in Ref. [8], since the dilaton coupling $g_{s}$ is related to the matrix model coupling constant, one can still think of "generalized" SL(2,R) conformal symmetry in which the string coupling is now regarded as a part of background fields that transform under the symmetry. The "generalized" conformal symmetry therefore transforms a matrix model at one value of the coupling constant to another. Note that, as pointed out in the previous section, the D0-brane in the dual frame has a description in terms of a domain-wall solution after the compactification on $\mathrm{S}^{8}$. So this is also related to the fact that in the domain-wall-quantum-field-theory (QFT) correspondence the choice of horosphere (the hypersurface of constant $u$ ) for the Minkowski vacuum corresponds to a

\footnotetext{
${ }^{3}$ At the ten-dimensional level, such $\operatorname{SL}(2, \mathbf{R})$ transformations act on the near-horizon supergravity D0-brane solution in such a way that the constant $Q$ in the harmonic function $H$ transforms as if it is a "field" on the world volume and then is set to a constant after the transformations [9].
} 
choice of coupling constant of a nonconformal QFT [13]. In the nonconformal case, the interpolation between the AdS Killing horizon (in the dual frame) and its boundary therefore corresponds to an interpolation between strong and weak coupling or vice versa.

The extreme limit of the probe D0-brane $[(m-q) \rightarrow 0]$ can be interpreted as M theory in the LCF, since in this case both the source and the probe D0-branes are in the Bogomol'nyi-Prasad-Sommerfield (BPS) limit. In this case, the functions $f$ and $g$ are simplified to

$$
f=\frac{1}{2} g_{s}^{-1}\left[\sqrt{m^{2}+g_{s}^{2}\left(\frac{\mu}{r}\right)^{7}\left(P_{r}^{2}+\frac{\vec{L}^{2}}{r^{2}}\right)}+m\right], \quad g=\frac{\vec{L}^{2}}{r^{2}},
$$

or in terms of the SYM theory variables

$$
\begin{gathered}
f=\frac{1}{2} \alpha^{\prime 1 / 2} g_{Y M}^{-2}\left[\sqrt{m^{2}+\frac{\alpha^{\prime-1} g_{Y M}^{6} N}{U^{7}}\left(P_{U}^{2}+\frac{\vec{L}^{2}}{U^{2}}\right)}+m\right], \\
g=\frac{\vec{L}^{2}}{U^{2}} .
\end{gathered}
$$

Unlike the case of a charged test particle in the nearhorizon Reissner-Nordström black hole background studied in Ref. [23], we do not let the mass of the source D0-brane bound state go to infinity, since the number $N$ of the D0branes in M theory on the LCF is kept finite. The fact that we are considering the near-horizon geometry of the D0-brane supergravity solution means that we are in the LCF of M theory, since the near-horizon geometry of the D0-brane supergravity solution is also the null reduction of the M-wave supergravity solution [21], which is interpreted as $M$ theory in the LCF. Taking $N$ to infinity, i.e., infinitely massive source D0-branes, corresponds to M theory in the IMF. Note that the IMF is defined as the limit in which $N$ and $R_{11}$ go to infinity such that the momentum $P_{11}=N / R_{11}$ also goes to infinity. Since $P_{11} \sim g_{Y M}^{-7 / 2} N^{1 / 4} U^{21 / 4}$ and $R_{11}$ $\sim g_{Y M}^{7 / 2} N^{3 / 4} U^{-21 / 4}$, one can take both $P_{11}$ and $R_{11}$ to infinity while taking $N \rightarrow \infty$, if $U$ is in the range of $g_{Y M}^{2 / 3} N^{-1 / 21} \ll U$ $\ll g_{Y M}^{2 / 3} N^{1 / 7}$. However, this range of $U$ is beyond the range of validity, Eq. (8), of the near-horizon solution (6). In other words, one cannot go to the IMF of M theory while keeping the parameters within the validity of the near-horizon solution (6). Anyway, the IMF means decompactification $\left(R_{11}\right.$ $\rightarrow \infty$ ) to 11 dimensions. So we should rather consider the M-wave solution in the case $N \rightarrow \infty$.

By expanding this Hamiltonian for an extreme D0-brane probe, one obtains a Hamiltonian of the form which is the sum of the nonrelativistic kinetic term for the probe and the velocity-dependent potential given by the sum of terms of the form $\sim \mathrm{v}^{2 n+2} / r^{7 n}\left(n \in \mathbf{Z}^{+}\right)$. This should reproduce the (M)atrix theory calculation of graviton-graviton scattering and its supergravity calculation reproduction of Ref. [21]. This is because the above action (18) is just the reduction of the action for the probe in the background of $D=11$ plane wave (describing the motion of the probe graviton in the background of the moving heavy source graviton) on a light- like circle to ten dimensions, i.e., $\mathrm{M}$ theory in the LCF. Note also that since M theory in the LCF has "Galilean" invariance (or is described by nonrelativistic quantum mechanics) in the transverse space, the above Hamiltonian for an extreme probe D0-brane has nonrelativistic structure. These above arguments, together with the fact that the generalized conformal mechanics of the probe D0-brane and the boundary $\mathrm{SU}(N)$ SYM theory [i.e., the (M)atrix model of M theory in the LCF] satisfy the same $\operatorname{SL}(2, \mathbf{R})$ symmetry, imply the equivalence between the (M)atrix model with finite $N$ and the generalized conformal mechanics of D0-branes.

Next, we consider the probe D0-brane moving in the background of the dual-frame metric of the source D0-brane bound state. Since the dual frame can be considered as a "holographic frame" describing supergravity probes [13], it is worthwhile to consider the case of the dual frame. In this frame, the near-horizon metric takes the $\operatorname{AdS}_{2} \times S^{8}$ form. So one should expect that the "generalized" conformal mechanics with the $\operatorname{SL}(2, \mathbf{R})$ symmetry can also be realized in the dual frame. The action for the probe D0-brane in the dual-frame background of the source D0-brane is given by Eq. (18) with the string-frame metric $G_{M N}^{s t r}$ replaced by the dual-frame metric $G_{M N}^{\text {dual }}$ through the relation $G_{M N}^{s t r}$ $=e^{2 \phi / 7} G_{M N}^{\text {dual }}$. So for the probe D0-brane in the dual frame, the dilaton factor $e^{-\phi}$ in Eqs. (18) and (19) [the dilaton factor $e^{-2 \phi}$ in Eqs. (20) and (24)] is replaced by $e^{-6 \phi / 7}\left[e^{-12 \phi / 7}\right]$.

Substituting the dual-frame near-horizon solution (10) into this general expression for the Hamiltonian corresponding to the dual frame, one finds that the Hamiltonian in the dual frame has the same form as the string-frame Hamiltonian (26) with the same $f$ and $g$ Eq. (27). In fact, in general the Hamiltonian describing probe D0-brane is independent of the near-horizon spacetime frame of the source D0-brane. So the dynamics of the probe D0-brane in the dual frame also has $\operatorname{SL}(2, \mathbf{R})$ symmetry with the same symmetry generators (30) and algebra (31) as the string-frame case.

As pointed out in the previous section, the source D0brane solution also has the (Hodge) dual description in terms of the eight-form field strength, whose magnetic charge now is carried by the source D0-brane. In this case, by compactifying the D0-brane solution on $\mathrm{S}^{8}$ one obtains a domainwall solution in $1+1$ dimensions $[33,13]$. This domain-wall solution solves the equations of motion of a $D=2 \mathrm{SO}(9)$ gauged maximal supergravity theory, which is an $S^{8}$ compactification of the type-IIA supergravity. This $\mathrm{SO}(9)$, which is the largest subgroup of the $\mathrm{SO}(16)$ and the isometry group of $S^{8}$ upon which the D0-brane is compactified, is also the $R$-symmetry group of the corresponding boundary $D=1$ QFT. In general, the $R$ symmetry of the supersymmetric QFT on the domain-wall world volume matches the gauge group, which is the isometry group of the compactification manifold, of the equivalent gauged supergravity [13].

Putting together all the above facts, namely (i) the generalized conformal mechanics of the probe D0-brane in the string-frame near-horizon background of the source D0brane is related to $\mathrm{M}$ theory in the LCF, (ii) the generalized 
conformal mechanics of the probe D0-branes in the string and the dual frames of the near-horizon source D0-branes are described by the same Hamiltonian, and (iii) upon dimensional reduction in $\mathrm{S}^{8}$ the bulk theory of the source D0-brane in the dual frame in the Hodge dual description (in terms of the eight-form field strength) is the $D=2 \mathrm{SO}(9)$ gauged maximal supergravity theory, one arrives at the speculation that $\mathrm{M}$ theory in the $\mathrm{LCF}$ is related to the $D=2 \mathrm{SO}(9)$ gauged maximal supergravity theory (i.e., a $D=2$ KaluzaKlein supergravity theory with domain-wall vacuum).
[1] G. 't Hooft, 'Dimensional reduction in quantum gravity,', gr-qc/9310026.

[2] L. Susskind, Phys. Rev. D 49, 6606 (1994).

[3] L. Susskind, J. Math. Phys. 36, 6377 (1995).

[4] J.M. Maldacena, Adv. Theor. Math. Phys. 2, 231 (1998).

[5] M.J. Duff, Class. Quantum Grav. 5, 189 (1988).

[6] G.W. Gibbons and P.K. Townsend, Phys. Rev. Lett. 71, 3754 (1993).

[7] M.J. Duff, G.W. Gibbons, and P.K. Townsend, Phys. Lett. B 332, 321 (1994).

[8] A. Jevicki and T. Yoneya, Nucl. Phys. B535, 335 (1998).

[9] A. Jevicki, Y. Kazama, and T. Yoneya, Phys. Rev. D 59, 066001 (1999).

[10] M.J. Duff and J.X. Lu, Nucl. Phys. B416, 301 (1994).

[11] H.J. Boonstra, B. Peeters, and K. Skenderis, Phys. Lett. B 411, 59 (1997)

[12] H. J. Boonstra, B. Peeters, and K. Skenderis, Nucl. Phys. B533, 127 (1998).

[13] H.J. Boonstra, K. Skenderis, and P.K. Townsend, J. High Energy Phys. 01, 003 (1999).

[14] M. Claudson and M.B. Halpern, Nucl. Phys. B250, 689 (1985).

[15] M.B. Halpern and C. Schwartz, Int. J. Mod. Phys. A 13, 4367 (1998).

[16] T. Banks, W. Fischler, S.H. Shenker, and L. Susskind, Phys. Rev. D 55, 5112 (1997).

[17] S. Weinberg, Phys. Rev. 150, 1313 (1966).
[18] B. de Wit, J. Hoppe, and H. Nicolai, Nucl. Phys. B305, 545 (1988).

[19] L. Susskind, "Another conjecture about M(atrix) theory," hep-th/9704080.

[20] N. Seiberg, Phys. Rev. Lett. 79, 3577 (1997).

[21] K. Becker, M. Becker, J. Polchinski, and A. Tseytlin, Phys. Rev. D 56, 3174 (1997).

[22] N. Itzhaki, J. M. Maldacena, J. Sonnenschein, and S. Yankielowicz, Phys. Rev. D 58, 046004 (1998),

[23] P. Claus, M. Derix, R. Kallosh, J. Kumar, P.K. Townsend, and A. Van Proeyen, Phys. Rev. Lett. 81, 4553 (1998).

[24] V. de Alfaro, S. Fubini, and G. Furlan, Nuovo Cimento A 34, 569 (1976).

[25] V.P. Akulov and A.I. Pashnev, Theor. Math. Phys. 56, 862 (1983).

[26] S. Fubini and E. Rabinovici, Nucl. Phys. B245, 17 (1984).

[27] J.M. Maldacena, Nucl. Phys. B (Proc. Suppl.) 68, 17 (1998).

[28] L. Susskind and E. Witten, "The holographic bound in Anti-de Sitter space,', hep-th/9805114.

[29] A.W. Peet and J. Polchinski, Phys. Rev. D 59, 065006 (1999).

[30] P.G.O. Freund and M.A. Rubin, Phys. Lett. 97B, 233 (1980).

[31] H. Lu, C.N. Pope, E. Sezgin, and K.S. Stelle, Nucl. Phys. B456, 669 (1995).

[32] H. Lu, C.N. Pope, E. Sezgin, and K.S. Stelle, Phys. Lett. B 371, 46 (1996).

[33] H. Lu, C.N. Pope, and P.K. Townsend, Phys. Lett. B 391, 39 (1997). 